\title{
AN EFFECTIVE USAGE PARAMETER CONTROL MODEL FOR CELL POLICING IN ATM NETWORKS
}

\author{
O. Osunade \\ Department of Computer Science, University of Ibadan, Nigeria \\ seyiosunade@gmail.com
}

\begin{abstract}
The primary role of traffic control procedures is to protect the network so that it can achieve the required network performance objectives and yield a high quality of service (QoS). To guarantee the QoS, the network implements traffic control using the parameters agreed upon at the start of data transfer, thus avoiding problems with degraded QoS before they occur. This paper presents an efficient model for policing cells transmitted over ATM networks. The model was simulated by a program written in Java. From several simulation test runs and subsequent analysis, it can be deduced that the model gives a minimal cell transfer rate at a minimum intercell period, while the cell delay variation tolerance is held constant. Optimal cell loss ratio is also achieved when the minimum intercell period is held constant and the cell delay variation tolerance is high. The results show minimal cell loss ratio has been achieved and the data throughput of the model is high.
\end{abstract}

\section{Index Terms}

Asynchronous transfer mode (ATM); cell transmission; traffic policing; leaky bucket algorithm

\section{Council for Innovative Research}

Peer Review Research Publishing System

Journal: INTERNATIONAL JOURNAL OF COMPUTERS \& TECHNOLOGY

Vol 12, No. 4

editor@cirworld.com

www.cirworld.com, www.ijctonline.com 


\section{INTRODUCTION}

ASYNCHRONOUS Transfer Mode (ATM) is a widely deployed data communication backbone technology because it supports a large array of technologies such as DSL, IP Ethernet, Frame Relay, SONET/SDH and wireless platforms. This transport medium is used to send data, video and voice at ultra high speeds within the core and at the edge of telecommunication systems. ATM allows easy integration with other technologies because of its enhanced management features that guarantee quality of service.

ATM services are still in demand though new technologies are emerging. ATM technology is still the only technology that can guarantee a certain and pre-defined quality of service. ATM offers low latency, making it suitable for time-sensitive or isochronous services such as video and voice while not being protocol or distance dependent. The growth of the Internet, electronic commerce and need for broadband access and multimedia content are driving the need for a reliable and efficient transport system such as provided by ATM.

ATM data transmission is based on parameter control with reference to values agreed upon before data transfer. There is a negotiation for facilities that will ensure reliable delivery of the data cells from the host source to their host destination. Two major techniques and their variants have been used to monitor the consistency and conformity of the data transfer parameters. However, high cell loss ratio and significant cell delay variation time are still problems that cannot be dealt successfully with by these existing techniques.

Thus this work develops a policing model for Usage Parameter Control (UPC) to manage data transmission on ATM networks. The specific objectives are to: develop a model that ensures integrity and reliability of end to end cell transmission; monitor and control traffic flow within the network; and, achieve traffic management automation that will efficiently react to the occurrence of significant situations and carryout the necessary real-time control operations.

\section{LITERATURE REVIEW}

Traffic control in an ATM network requires that users declare the traffic characteristics of a cell stream when connections are being setup and the network assigns the resources for the communication if it judges that these resources are available. If the assignment is impossible, the connection is rejected. During communication, the network monitors the conformity between the declared traffic and the characteristics of the actual cell stream at the entrance of the network [1]. If the network finds nonconformities in the transmission, it imposes a penalty on the user. The control system assigning resources and judging rejection or acceptance of the connection is the connection admission control (CAC), and the control system monitoring the cell stream and its violation is the usage parameter control (UPC). An additional role of traffic control is to optimize the use of network resources for the purpose of achieving realistic network efficiency. According to [2] the following functions form a framework for managing and controlling traffic in ATM networks: Network Resource Management; Connection Admission Control (CAC); User Parameter Control (UPC).

Network resource management is responsible for the allocation of network resources in order to separate traffic flows according to different service characteristics, to maintain network performance and to optimize resource utilization. This function is mainly concerned with the management of virtual paths in order to meet QoS requirements.

With relation to traffic control, virtual path connections (VPCs) can be used to simplify CAC; implement a form of priority control by segregating traffic types requiring different quality of service; distribute messages efficiently for the operation of traffic control schemes; and aggregate user-to-user services such that the usage parameter control can be applied to the traffic aggregate.

VPC's play a key role in network resource management. By reserving capacity on VPC's, the processing required to establish individual VCC's is reduced. Individual VCC's can be established by making simple connection admission decisions at nodes where VPC's terminated. Strategies for the reservation of capacity on VPC's will be determined by the trade -off between increased capacity costs and reduced control costs. These strategies are left to the operator.

Where VCC's within a VPC require a range of quality of service, the VPC is provided with a QoS suitable for the most demanding VCC's carried e.g. if one of the VCC's within a VPC requires the allocation of the peak cell rate equal to a significant proportion of the VPC capacity, then the assurance of the QoS of this VCC may require that all other VCCs within this VPC also have an allocation of the peak capacity. The cell loss priority bit may be used to distinguish the two levels of cell loss on a VPC.

Connection Admission Control is defined as the set of actions taken by the network at the call set-up phase or during call re-negotiation phase in order to establish whether a VCC or a VPC can be accepted or rejected [3], [4]. A connection request is accepted only when sufficient resources are available to establish the call through the whole network at its required quality of service and to maintain the agreed quality of service of existing calls. This applies also to re-negotiating of connection parameters within a given call.

In a B-ISDN environment, a call can require more than one connection, for instance for multimedia or multi-party services such as video-telephony/video-conferencing. In this case, CAC procedures should be performed for each VPC or VCC. In the case of an on-demand service, the connection-establishment procedures will enable the CAC to derive at least the following types of information: source traffic characteristics or required QoS class.

In the case of a permanent or reserved service, e.g. using a permanent VPC or permanent VCC, this information is indicated with an appropriate Operations And Maintenance (OAM) procedure either through an online e.g. signalling or offline e.g. service order procedure. 
Traffic characteristics are described by the parameters listed below, some of which are mutually dependent [5], [6]: Minimum cell-rate (MCR), Peak cell-rate (PCR), Sustained (Average) cell-rate (SCR), Initial cell-rate (ICR), Burst Tolerance (BT), Cell Delay Variation Tolerance (CVDT), Source type (e.g. telephone, multimedia).

Usage Parameter Control (UPC) function is performed at the User-Network-Interface (UNI). Pardeepinit and Jittawiriyanukoon [4] defined UPC as the set of actions taken by the network to monitor and control traffic in terms of conformity with the agreed traffic contract and cell routing validity at the user access. The main purpose is to protect network resources from malicious or unintentional acts that could affect the QoS of other established connections [7]. It does this by detecting violations of the negotiated parameters and taking appropriate action as indicated in Table 1.

Table 1: Options available to UPC for controlling violating cells

\begin{tabular}{|l|l|}
\hline $1 . \quad$ Violation & Action taken \\
\hline Discarding cells & Violating cells are dropped \\
\hline Tagging cells & CLP bit is set so that cells can be discarded later if necessary \\
\hline Dropping connection & The complete connection is dropped \\
\hline
\end{tabular}

UPC applies to both user VCCs/VPCs and signaling virtual channel. The monitoring of usage parameters for VCCs and VPCs is done by checking the validity of VPI and VCI and monitoring the traffic entering the network from active VPCs and VCCs in order to ensure that parameters agreed upon are not violated.

A policing algorithm should always be able to detect and quickly respond to any traffic violation in the network. It should also not take any action on traffic that conforms, i.e. traffic that doesn't violate the traffic contract negotiated at call set-up. Several policing algorithms have been proposed to regulate traffic flows in a network by controlling the sources' traffic parameters. The algorithms can be divided into two main groups:

A Window based mechanisms, which limits the number of cells in a time window. These groups consider algorithms in which fixed or variable time windows limit the number of cell arrivals. Examples of window algorithms are schemes like jumping window (JW), Triggered Jumping Moving Window (TJMW) and the exponentially weighed moving average (EWMA)

A Leaky bucket (LB) increments a counter for each cell arrival and periodically decrements this counter. The Leaky Bucket method is generally agreed to achieve the best performance of the methods studied. Its advantages are simplicity and flexibility. The idea behind the Leaky Bucket is that, when a cell arrives in the network, it must get a token from the token pool. If there are any tokens available in the token pool on cell arrival, the cell takes one token and one decreases the token pool size. The tokens are generated at a constant rate and the token pool is like a virtual finite queue. Cell arrivals are discarded (or tagged) when the token pool is empty.

The algorithm can be schematically described in its simplest form by the following:

1. increasing a counter $C$ by 1 every $T$ seconds up to $M$ if there are no cell arrivals;

2. decrement the counter $\mathrm{C}$ by 1 for each transmitted cell;

3. drop (or tag) cells when $\mathrm{C}=0$.

$M$ represents the maximum burst size, while $1 / T$ is the peak cell rate of the traffic source being observed.

Using the LB scheme can police both VPs and VCs. When VP policing is performed, the only measure of interest is the aggregate bit rate offered to the VPs, which can usually be easily policed; therefore, the contents of each VC multiplexed in a VP and their source characteristics are ignored for policing purposes. However, when policing is executed on a VC basis, it becomes more complicated to control the sources' traffic parameters and, as in this case, the sources have to be characterized by at least their peak and mean cell rates.

The following parameters have been used to evaluate ATM networks

Cell Delay Variation (CDV): In ATM networks, the cell delay experienced by cells of the same connection can vary. This is called cell delay variation (CDV) and is caused mainly by buffering at ATM switching nodes. Since CDV can change the traffic characteristics of sources in the network, the bandwidth required by those sources can also change. Therefore, UPC should take CDV into account when deciding whether cell arrivals are violating the respective traffic contract negotiated at call set-up. In this case, the maximum allowed CDV should be an extra parameter to be included in the traffic contract. When UPC takes CDV into account, it introduces tolerances in the amount of traffic from a source that conforms to the corresponding traffic contract. It is thus possible that some cells that are really violating the traffic contract are not discarded.

Cell Loss Ratio (CLR): This is the ratio of discarded cells to cells that are successfully transmitted. Mathematically represented as,

$$
\mathrm{CLR}=\frac{\text { discarded cells }}{\text { transmitted cells }}
$$


CLR is defined in the ATM Forum's "Traffic Management Specification - Version 4.0" as a method of using OAM cells for in-service measurement of cell loss. OEM cells with a count of the number of user information cells transmitted since the last OAM cell are inserted into the cell stream. The receiver keeps track of the number of user information cells transmitted (Nt) and received (Nr). The Cell Loss Ratio calculation is:

$$
\mathrm{CLR}=(\mathrm{Nt}-\mathrm{Nr}) / \mathrm{Nt} \text { if } \mathrm{Nt}-\mathrm{Nr}>0
$$

Peak Cell Rate (PCR): The Peak Cell Rate, PCR is the maximum instantaneous rate at which the user will be permitted to transmit. This parameter is the inverse of the minimum intercell period. Over a longer period the average rate at which a source is allowed to send is known as the Sustainable Cell Rate (SCR).

$$
\mathrm{PCR}=1 / \mathrm{T}
$$

where T- minimum intercell period

Burst Tolerance (BT): The Burst Tolerance (BT) makes use of the leaky bucket algorithm to limit the maximum burst size (MBS) that may be transmitted at the PCR. All cells entering the network are placed in this bucket, which is drained at the SCR. The depth of the bucket is:

$$
\mathrm{BT}=(\mathrm{MBS}-1)(1 / \mathrm{SCR}-1 / \mathrm{PCR})
$$

\section{PROPOSED MODEL}

The proposed scheme has an edge over earlier implementations because it allows for the checking of the validity of Virtual Path Identifier (VPI) and Virtual Channel Identifier ( $\mathrm{VCl}$ ) values. It also allows the proper monitoring of traffic entering a network from each active VCC and VPC in order to ensure that parameters agreed upon are not violated.

The model developed is based on the Generic Cell Rate Algorithm (GCRA) which is a variant of the leaky bucket algorithm. GCRA takes into account the cell delay variation tolerance and determines the cells that are to be discarded. The proposed model is shown in Figure 1a with the following key components: Source, Amofin (cell police), Regulator and GCRA.

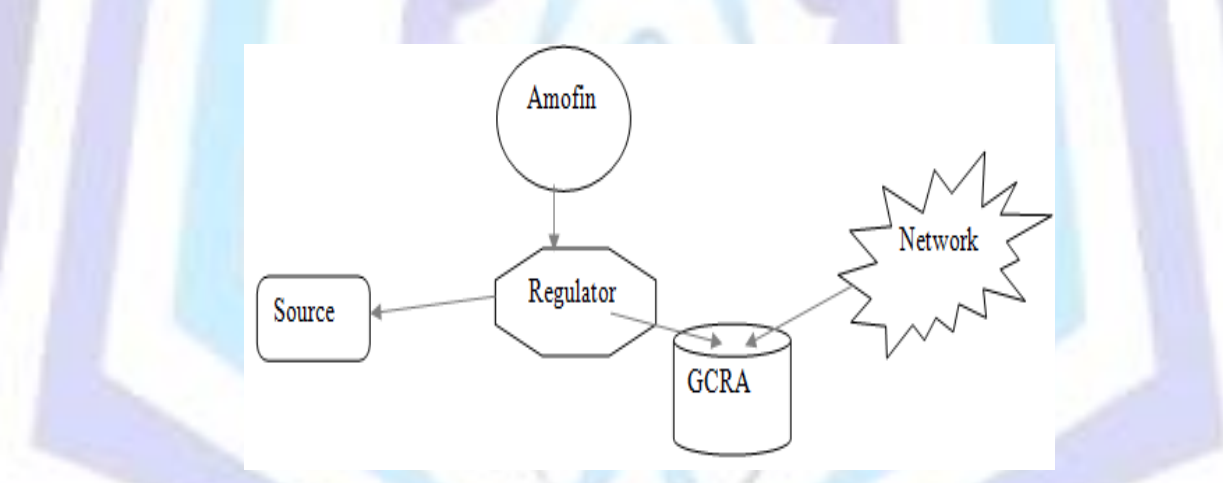

Figure 1a: Schematic diagram of the ATM Policing Architecture

Source: this is where the cells are generated within the network. A contract is setup before end -to-end cell transmission can take place.

Amofin: this is the ATM cell transmission police that enforces and ensures that a cell transmitted by the source does not violate the contract established at connection setup. If violation occurs it passes execution to leaky bucket.

Regulator: this is a temporary buffer where the actions/operations of the source and police are synchronized.

\section{Generic Cell Rate Algorithm (GCRA):}

this takes into account the cell delay variation tolerance and determines the cells that are to be discarded.

After the connection setup by the CAC, the source sends a notification to the regulator to send a cell. Amofin then checks the regulator for a send cell notification, if the notification is confirmed; Amofin then records the parameters of the first cell. These parameters are then taken as those necessary for cell transmission. The Amofin then negates the content of the regulator. The regulator waits for a time period less or equal to the minimum intercell period $(\mathrm{T})$ and the cell arrival counter is incremented until its value is equal to the maximum burst size (MBS). The transmission cells are then passed through the GCRA, which then decides if transmitted cells are conforming or non- conforming. 


\section{METHODOLOGY}

In this work, the proposed model was tested by simulation using a Java program. Various parameters involved in data transmission on an ATM network were investigated. The simulation program follows the algorithm shown in Figure $1 \mathrm{~b}$ below.

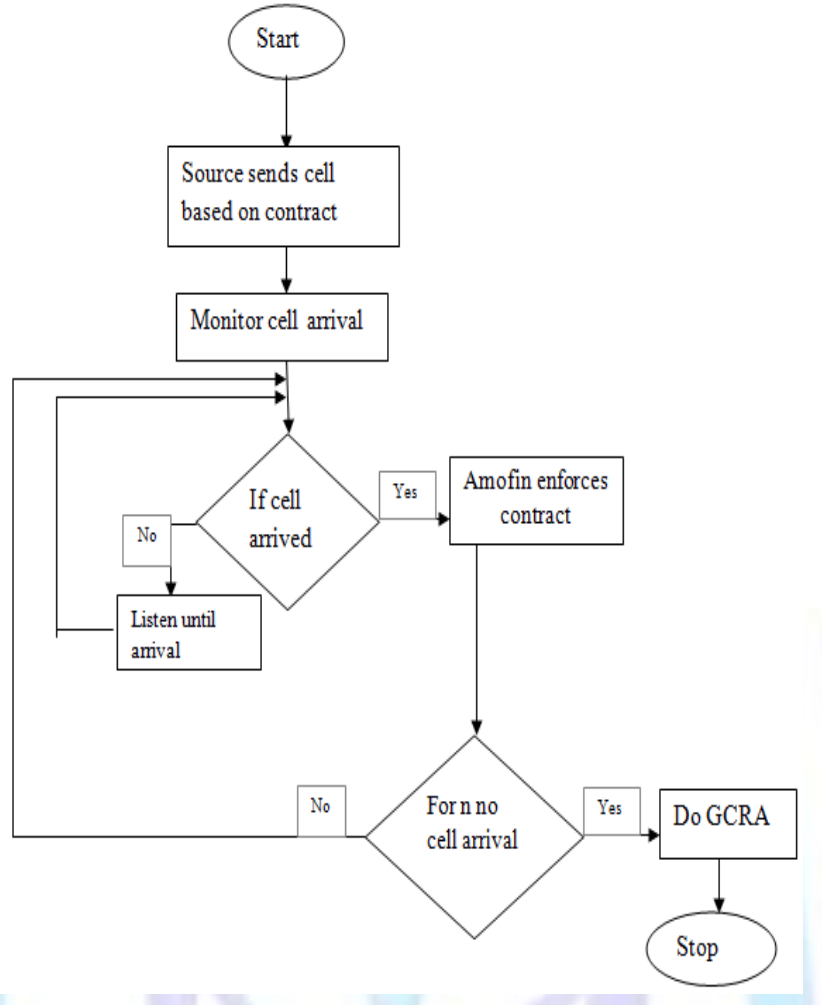

Figure 1b: Flowchart of algorithm for the proposed model

\section{RESULTS AND ANALYSES}

The simulation of the various performance parameters under study was analyzed with a view to getting real-time effects and characteristics of the model when placed under certain conditions and to get the best working condition. The performance analysis of the model was carried out by varying parameters such as Cell Delay Variation tolerance (CDVT), Maximum Burst Size (MBS), Peak Cell Rate (PCR) against Cell Loss Ratio (CLR).

\section{Analysis of Maximum Burst Size (MBS)}

This is the maximum cell capacity of the leaky-bucket algorithm as implemented in the simulation. Shown in Table $2 a$ and $2 \mathrm{~b}$ are some of the values generated by the simulation program.

Table 2a: MBS against CLR values at transfer rate of 10 cells

\begin{tabular}{|c|c|c|c|c|}
\hline $\mathrm{T}=1 / \mathrm{PCR}$ & CDVT & CLR & MBS & No. of Cells \\
\hline 100 & 100 & 0.8 & 100 & 10 \\
\hline 100 & 100 & 0.8 & 120 & 10 \\
\hline 100 & 100 & 0.8 & 140 & 10 \\
\hline 100 & 100 & 0.8 & 200 & 10 \\
\hline 100 & 100 & 0.8 & 400 & 10 \\
\hline
\end{tabular}


Table 2b: MBS against CLR values at transfer rate of 100 cells

\begin{tabular}{|c|c|c|c|c|}
\hline T=1/PCR & CDVT & CLR & MBS & No. of Cells \\
\hline 100 & 100 & 0.89 & 100 & 100 \\
\hline 100 & 100 & 0.89 & 120 & 100 \\
\hline 100 & 100 & 0.89 & 140 & 100 \\
\hline 100 & 100 & 0.89 & 200 & 100 \\
\hline 100 & 100 & 0.89 & 400 & \\
\hline
\end{tabular}

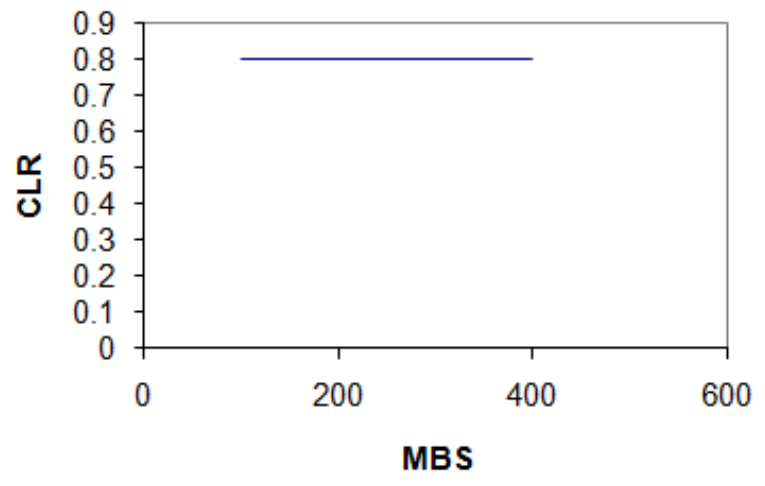

Figure 2a: Graph of MBS against CLR at transfer of 10 cells

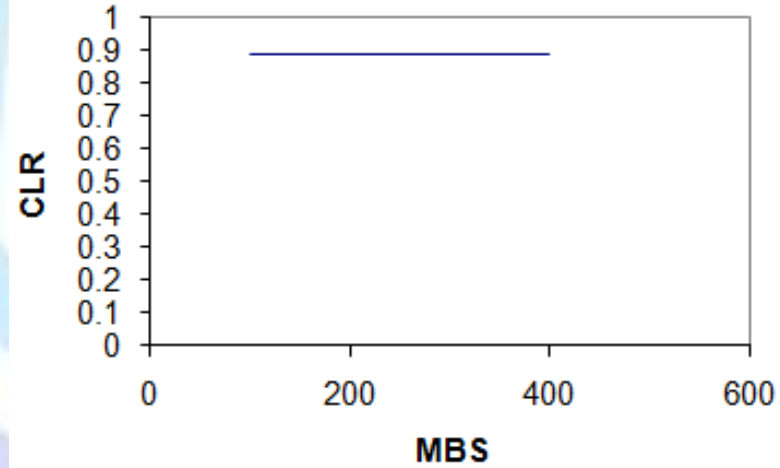

Figure 2b: Graph of MBS against CLR at transfer of 100 cells

From Figures $2 a$ and $2 b$ respectively which were plotted from the values given in Tables $2 a$ and $2 b$, it can be observed that the variation of the MBS does not yield significant change in the value of CLR irrespective of the number of cells transmitted over the network. Note that the minimum intercell period $(T)$ and the Cell Delay Variation Tolerance (CDVT) were held constant.

\section{Analysis of Peak Cell Rate (PCR)}

PCR is the maximum instantaneous rate at which the user will be permitted to transmit. This parameter is the inverse of the minimum intercell period (i.e. 1/T). Shown in Tables $3 a$ and $3 b$ are some of the values generated by the simulation program: 
Table 3a: PCR against CLR values at transfer rate of 10 cells

\begin{tabular}{|c|c|c|c|}
\hline $\mathrm{T}=1 / \mathrm{PCR}$ & CDVT & CLR & No. of Cells \\
\hline 10 & 100 & 0.1 & 10 \\
\hline 20 & 100 & 0.4 & 10 \\
\hline 30 & 100 & 0.6 & 10 \\
\hline 40 & 100 & 0.7 & 10 \\
\hline 50 & 100 & 0.7 & \\
\hline
\end{tabular}

Table 3b: PCR against CLR values at transfer rate of 100 cells

\begin{tabular}{|c|c|c|c|}
\hline T=1/PCR & CDVT & CLR & No. of Cells \\
\hline 10 & 100 & 0.89 & 100 \\
\hline 20 & 100 & 0.94 & 100 \\
\hline 30 & 100 & 0.96 & 100 \\
\hline 40 & 100 & 0.97 & 100 \\
\hline 50 & 100 & 0.97 & \\
\hline
\end{tabular}

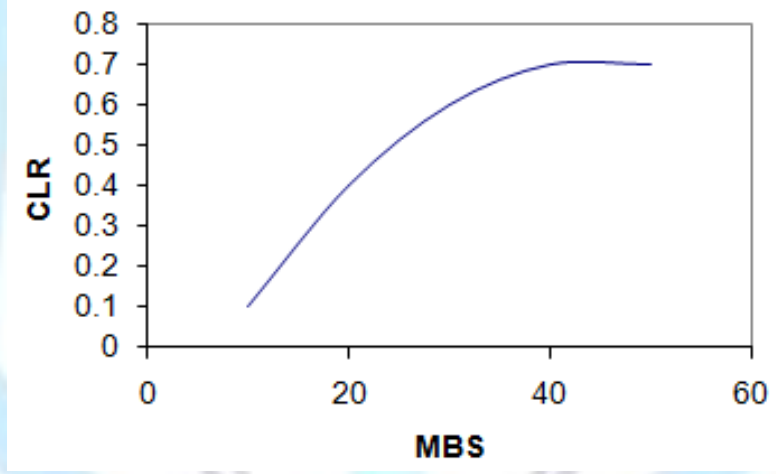

Figure 3a: Graph of PCR against CLR at the transfer rate of 10 cells

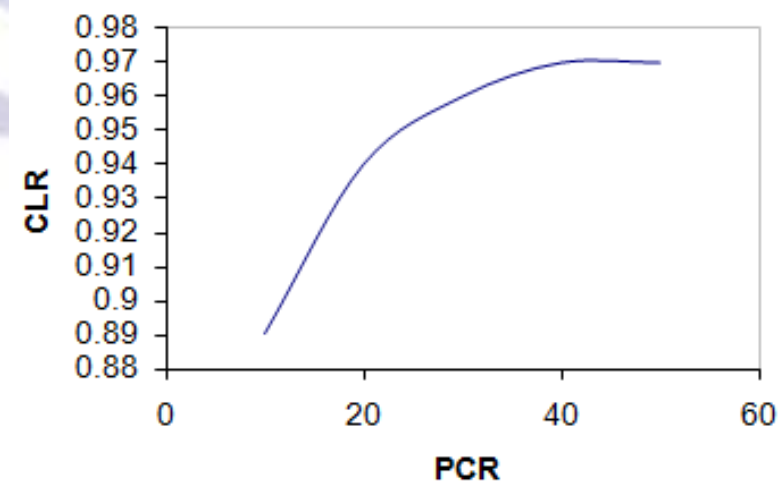

Figure 3b: Graph of PCR against CLR at the transfer rate of 100 cells

From Figure $3 a$, it can be observed that increase in the minimum intercell period leads to a sharp and drastic decline in the cell loss ratio when the cell transfer rate is set at 10 . On the other hand, from Figure $3 \mathrm{~b}$ increase in the minimum intercell period does not produce a significant change in cell loss ratio when the cell transfer rate is set at 100. 


\section{Analysis of Cell Delay Variation Tolerance (CDVT)}

CDVT is the maximum allowable delay variation that can occur during cell transmission.

Tables $4 a$ and $4 \mathrm{~b}$ show some of the values obtained from the simulation program:

Table 4a: CDVT against CLR values at transfer rate of 10 cells

\begin{tabular}{|c|c|l|c|}
\hline T=1/PCR & CDVT & CLR & No. of Cells \\
\hline 100 & 50 & 0.9 & 10 \\
\hline 100 & 100 & 0.8 & 10 \\
\hline 100 & 200 & 0.7 & 10 \\
\hline 100 & 400 & 0.5 & 10 \\
\hline 100 & 800 & 0.2 & 10 \\
\hline 100 & 1200 & 0.1 & 10 \\
\hline
\end{tabular}

Table 4b: CDVT against CLR values at transfer rate of 100 cells

\begin{tabular}{|c|c|c|c|}
\hline T=1/PCR & CDVT & CLR & No. of Cells \\
\hline 100 & 50 & 0.99 & 100 \\
\hline 100 & 100 & 0.98 & 100 \\
\hline 100 & 200 & 0.97 & 100 \\
\hline 100 & 400 & 0.95 & 100 \\
\hline 100 & 800 & 0.91 & 100 \\
\hline 100 & 1200 & 0.87 & 100 \\
\hline
\end{tabular}

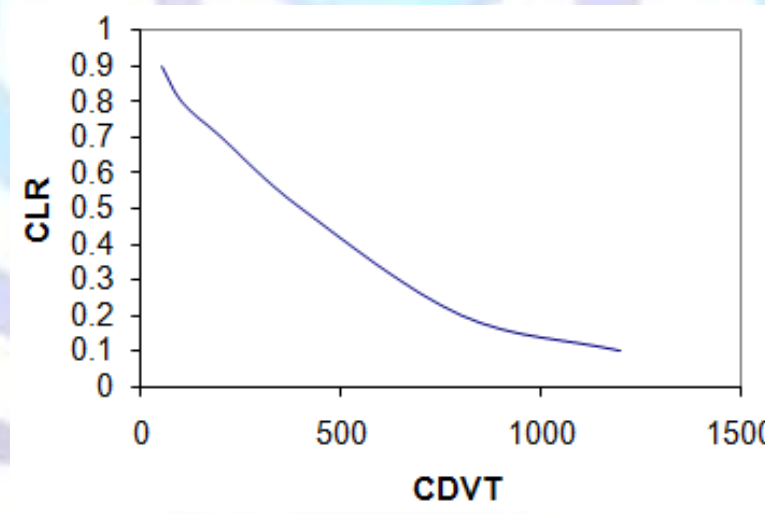

Figure 4a: Graph of CDVT against CLR at the transfer rate of 10 cells 


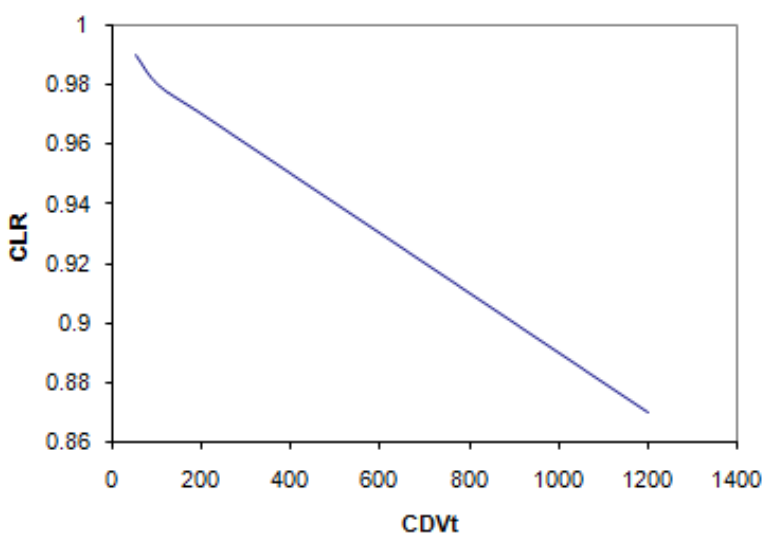

Figure 4b: Graph of CDVT against CLR at the transfer rate of 100 cells

From Figure $4 \mathrm{a}$, it can be observed that increase in CDVT at a constant minimum intercell period $(T)$, there is drastic increase in cell loss ratio with the cell transfer rate set at 10 . While in Figure $4 \mathrm{~b}$ there is a slow convergence of the cell loss ratio to a maximum with the cell transfer rate set at 100 .

\section{CONCLUSION}

ATM networks have a policing mechanism in place to control and monitor the data traffic based on cell transmission. The quality of service deficiencies in existing cell transmission management techniques was addressed by the proposed policing model. The model implemented here discards non-conformant cells so as to lead to a higher quality of service, minimal cell loss and makes congestion on the data network almost impossible. The model allows a minimal cell transfer rate at a minimum intercell period, while the cell delay variation tolerance is held constant. Optimal cell loss ratio is also achieved when the minimum intercell period is held constant and the cell delay variation tolerance is high.

\section{ACKNOWLEDGEMENTS}

The author would like to acknowledge O. B. Oginni and G. A. Aderounmu for the initial experimental runs of the simulation carried out.

\section{REFERENCES}

[1] Makarechian, M. and Malcolm, N. J. 1997. Testing Policing in ATM Networks. Hewlett-Packard Journal Arcticle 11, 1-8.

[2] Black U.D. (1995) ATM-foundation for Broadban Networks, Published by Prentice Haii, USA.

[3] Saito, H. (1994). In Teletraffic Technologies in ATM Networks . Artech House, USA ISBN: 0890066221.

[4] Pakdeepinit, P. and Jittawiriyanukoon, C. 2002. Performance Comparison of ATM Policing Mechanisms with Telecommunications traffic. Australian Journal of Technology 6(2): 61-69.

[5] Handel R., Manfred H., Schroder S. (1994). . In ATM Networks: Concepts, Protocols, Applications . AddisonWesley Publishing Company Inc.

[6] Ferguson P., Huston G. (1998). . In Quality of Service: Delivering QoS on the Internet and in Corporate Networks. John Wiley and Sons, Inc.

[7] Battaglia, L. (2001) The Usage Parameter Control Issue in ATM Networks, Publisher Books on Demand, pg 1159, ISBN 978-3831110551 